\title{
Biallelic TMEM251 variants in patients with severe skeletal dysplasia and extreme short stature
}

\author{
Noor Ain ${ }^{1}$, Niaz Muhammad ${ }^{1}$, Mehdi Dianatpour ${ }^{2}$, Marta Baroncelli $^{3}$, Muddassar Iqbal ${ }^{4}$, \\ Mohammad A. Farazifard ${ }^{2}$, Ihtisham Bukhari ${ }^{4}$, Sufian Ahmed ${ }^{1}$, Massoumeh Hajipour ${ }^{5}$, \\ Zahra Tabatabaie ${ }^{5}$, Hamidreza Foroutan ${ }^{5}$, Ola Nilsson ${ }^{3}$, Mohammad Ali Faghihi ${ }^{5}$, Outi \\ Makitie $^{3}$, and Sadaf $\mathrm{Naz}^{4}$ \\ ${ }^{1}$ University of the Punjab Quaid-i-Azam Campus \\ ${ }^{2}$ Shiraz University of Medical Sciences \\ ${ }^{3}$ Karolinska Institute \\ ${ }^{4}$ University of the Punjab \\ ${ }^{5}$ Persian BayanGene Research and Training Center
}

June 10, 2020

\begin{abstract}
Skeletal dysplasias are a heterogeneous group of disorders ranging from mild to lethal skeletal defects. We investigated two unrelated families with individuals presenting with a severe skeletal disorder. In family NMD02, affected individuals had a dysostosis multiplex-like skeletal dysplasia and severe short stature $(<-8.5 \mathrm{SD})$. They manifested increasing coarse facial features, protruding abdomens and progressive skeletal changes, reminiscent of mucopolysaccharidosis. The patients gradually lost mobility and the two oldest affected individuals died in their twenties. The affected child in family ID01 had coarse facial features and severe skeletal dysplasia with clinical features similar to mucopolysaccharidosis. She had short stature, craniosynostosis, kyphoscoliosis and hip-joint subluxation. She died at the age of 5 years. Whole-exome sequencing identified two homozygous variants c.133C > T; p.(Arg45Trp) and c.215dupA; p.(Tyr72Ter) respectively, in the two families, affecting an evolutionary conserved gene TMEM251. Immunofluorescence and confocal studies on Human Osteosarcoma cells indicated that TMEM251 localized to the Golgi complex and plasma membrane. However, p.Arg45Trp mutant TMEM251 protein was targeted less efficiently to the membranes and the localization was punctate. Tmem251 knockdown by siRNA induced dedifferentiation of rat primary chondrocytes. Our work implicates TMEM251 in the pathogenesis of a novel disorder and suggests its potential function in chondrocyte differentiation.
\end{abstract}

\section{Introduction}

Skeletal dysplasias are a heterogeneous group of inherited disorders of mostly monogenic etiology. They affect skeletal development and lead to variable skeletal anomalies, often with short stature.(Krakow, 2015; Mortier et al., 2019) Some forms are associated with a spectrum of metabolic disturbances and extra-skeletal manifestations in the context of complex syndromes. The manifestations can include, but are not limited to hepatosplenomegaly, cardiomyopathy and pulmonary dysfunction.(Longo et al., 2013; Saenger, Czernichow, Hughes, \& Reiter, 2007) Many of the metabolic disturbances observed in this group of disorders arise from perturbations of pathways involving modifications of proteins and lipids.(Sparks \& Krasnewich, 2017) These modifications are important for stability or function of molecules including proteins which function in several tissues including cartilage and bone.(Stanley, 2011) For example, many skeletal disorders with prominent extra-skeletal manifestations involve defects in glycosylation.(Coman, Irving, Kannu, Jaeken, \& Savarirayan, 2008; Kranz et al., 2007) 
Several metabolic disorders with severe skeletal consequences have been identified and delineated. These include lysosomal storage disorders, mucopolysaccharidosis (OMIM\#309900, OMIM\#253200, OMIM\#25300), mucolipidosis (OMIM\#252600, OMIM\#252605), and congenital disorders of glycosylation (OMIM\#212065), among others. These disorders can be inherited in an autosomal recessive, autosomal dominant, X-linked recessive or X-linked dominant manner. The multisystemic defect due to error in metabolism in these and most other related disorders is progressive and severity of the disorder increases with age. Cognition and hearing ability can also be affected. In extreme cases, multi-organ failure and early death can occur.(Ballabio \& Gieselmann, 2009; Eklund \& Freeze, 2006) Although the molecular bases of many skeletal disorders are known, a significant number of others remain to be elucidated.

In this study, we explored the genetics of severe skeletal dysplasia in individuals from Pakistan and Iran. Patients in both families had features similar to mucopolysaccharidosis.

\section{Material and Methods}

\section{Editorial Policies and Ethical Considerations}

The research on human subjects was approved by Institutional Review Board of School of Biological Sciences, University of the Punjab, Lahore, Pakistan and ethics committee of Persian Bayangene Research and Training Institute, Shiraz, University of Medical Sciences, Iran. All participants or the parents for minor children provided written informed consent. Animals were handled according to the protocol approved by the Bioethical Committee of School of Biological Sciences, University of the Punjab, Lahore, Pakistan and Bioethical Committee of Karolinska Institutet, Stockholm, Sweden.

\section{Subjects}

We identified two consanguineous families NMD02 and ID01 (BY0084) (Figure 1), in which affected individuals had a Mucolipidosis II alpha/beta like syndrome (OMIM\#252500). Clinical analyses involved complete blood counts and radiographs for two affected individuals III:9 and III:11 of family NMD02 and affected child IV:5 of ID01. Morning urine samples were collected from three affected III:9, III:10, III:11 and two unaffected individuals II:3, III:3 of family NMD02 for qualitative measurements of glycosaminoglycan levels by Berry spot test. Briefly the urine sample was applied to a filter paper and air dried. Then toluidine blue $\mathrm{O}$ was applied and development of purple color was regarded as presence of GAGs in urine. Lysozyme enzymes from dried blood were measured for individual IV:5 of family ID01. Blood samples were collected from all participants for genetic studies and the DNA was extracted by a standard protocol.

\section{Molecular Analyses}

For family NMD02, fluorescently labeled microsatellite markers located close to the respective genes (Table S1) were genotyped to exclude linkage of the disorder to GNPTAB (OMIM\#607840), GNPTG(OMIM\#607838), IDUA (OMIM\#611542) and ARSB(OMIM\#611542). Samples from two affected individuals III:5 and III:9 from family NMD02 were chosen for exome sequencing using the Agilent human V5 kit (51Mb), (Agilent Technologies, Santa Clara, CA, USA). Paired-end reads were obtained at 50X average target coverage on an Illumina Hi-Seq 2500 sequencer (Otogenetics, Norcross, GA, USA). Samples from parents and the affected child were assessed by exome sequencing for family ID01.

For family NMD02, we analyzed the exome data for identification of variants unique to each patient, as well as for those identical to both affected individuals. Variants in the known genes for Mucolipidosis and Mucopolysaccharidosis (Table S1) were also checked. All homozygous variants and compound heterozygous variants in the exome data were examined further after filtering. Variants with allele frequencies of less than 0.01 in all public databases were retained. Further choice was made for exonic missense, nonsense frameshift and in-frame insertion or deletion variants. Synonymous and intronic variants affecting splice sites or creating new splice sites, were also considered.

Exome variant call files for two affected individuals of NMD02 were analyzed with Agilevariant as described (Carr et al., 2013) to display regions of homozygosity in individual data and those common to both samples 
were identified (Supplementary Figure 1). Exome coverage data were examined to identify incompletely sequenced exons in one or both of the samples. Those exon located within regions of shared homozygosity were sequenced after amplification with specific primers (Table S2) using a standard PCR protocol or a protocol for GC-rich amplification.(Naz \& Fatima, 2013)

Data for the three samples of family ID01 was analyzed by Burrow Wheel Aligner (BWA) (Li \& Durbin, 2009), GATK (McKenna et al., 2010) and ANNOVAR.(Wang, Li, \& Hakonarson, 2010)

\section{Computational analyses}

We utilized wAnnovar for analyzing the exome data in order to prioritize variants. The output file of this analysis included frequency of each variant in large public databases as well as predictions of pathogenicity of missense variants by multiple software. Pathogenicity scores for all missense variants according to REVEL were examined by downloading individual chromosomal files. The computational tools Predictprotein and Protfun, were used to predict the secondary structure, identify motifs and suggest possible protein function. Multiple sequence alignments of the protein sequences were performed using Clustal Omega.

\section{cDNA synthesis}

We extracted RNA from blood (human) or brain, liver and kidney (mouse) using Tri Reagent (Thermo Fisher Scientific). The cDNA libraries were prepared using RevertAid First Strand cDNA Synthesis Kit (Thermo Fisher Scientific) primed with a Poly T primer. TMEM251 cDNA was amplified using gene specific primers (Table S3). The cDNA were cloned by $\mathrm{T} / \mathrm{A}$ cloning into $\mathrm{pTZ57R} / \mathrm{T}$ vector (Thermo Fisher Scientific) and sequence verified.

\section{TMEM251 GFP fusion constructs}

The plasmid pACGFP-N1 (Clontech, California, USA) was used for obtaining TMEM251 fused to GFP at its C-terminal. TMEM251 cDNA cloned in $\mathrm{pTZ} 57 \mathrm{R} / \mathrm{T}$ vector was amplified using gene specific primers with flanking recognition sequences for restriction enzymes BamH1 andEcoR1 (Table S4). Ligation of restriction digested PCR amplified TMEM251 cDNA and similarly cut vector was completed using T4 DNA ligase. The recombinant plasmid was transformed into competent $E$. coli . The bacteria were plated on LB agar containing $100 \mu \mathrm{g} / \mathrm{ml}$ of kanamycin and grown at $37^{\circ} \mathrm{C}$ overnight. Colonies were picked and plasmids were isolated using Qiagen minikit (Qiagen, Hilden, Germany). Insertion of cDNA into vector was confirmed by sequencing. Site directed Ligase Independent Mutagenesis was carried out to introduce the c.133C $>\mathrm{T}$ p.(Arg45Trp) variant into the wild-type TMEM251 cDNA cloned in pACGFP-N1 as described.(Chiu, March, Lee, \& Tillett, 2004) Briefly, an inverse PCR was done in two reactions to amplify the template plasmid containing the cloned wild-type TMEM251 open reading frame using two tailed long and two short primers (Table S4).

\section{Transfections, Immunocytochemistry and confocal microscopy}

Human Osteosarcoma cells (Sigma-Aldrich, Darmstadt, Germany) were grown in DMEM (Sigma-Aldrich) complete medium to a confluency of 70 percent and then C-terminal GFP fused wild-type or mutant TMEM251 was transiently transfected using Lipofectamine 3000 (Thermo Fisher Scientific) following manufacturer's protocol. Cells were fixed in $4 \%$ paraformaldehyde and blocked using $5 \%$ anti-goat serum in $0.1 \%$ triton X100/ PBS. Primary antibodies PDI mouse monoclonal antibody (Enzo Life Sciences, Nassau, NY) for Endoplasmic reticulum and Giantin mouse monoclonal antibody for Golgi complex (Enzo Life Sciences) were diluted in blocking medium to a final dilution of 1 in 100. Secondary staining was completed by using Goat anti-Mouse IgG Cross-Adsorbed Secondary Antibody (Thermo Fisher Scientific) diluted in blocking medium to a final dilution of 1 in 2000. Coverslips were mounted onto slides using Vectashield ${ }^{\circledR}$ hardset mounting media containing DAPI (Vector Laboratories, California, USA). Intracellular localization of both the wild-type and the mutant TMEM251 GFP fusions (GFP fused at C-terminal) were observed by confocal imaging of the transfected cells after immunostaining. Human Osteosarcoma cells were examined with LSM 700 Laser Scanning Microscope (Carl Zeiss, Oberkochen, Germany) equipped with Zeiss AxioCam camera (Carl Zeiss) and ZEN 2011 digital imaging software (Carl Zeiss). 


\section{Chondrocyte isolation and culture}

Proximal tibias and distal femurs were dissected from 3 to 5 day old rats (Charles River Laboratory, Wilmington, MA, USA). These were digested with $0.3 \%$ collagenase type IA (Sigma-Aldrich) in DMEM/F12 aseptically, as described.(Spath, Andrade, Chau, Baroncelli, \& Nilsson, 2018) Briefly, dissected cartilage pieces were washed twice in PBS with $1 \%$ penicillin/streptomycin, $50 \mathrm{ng} / \mathrm{ml}$ fungizone (both from Thermo Fisher Scientific), next incubated with 0.1\% EDTA (Sigma-Aldrich) in PBS for 15 minutes and $0.125 \%$ trypsin (Thermo Fisher Scientific) in PBS for 30 minutes, all steps at $37^{\circ} \mathrm{C}$ while shaking. Cartilage pieces were washed twice in PBS with $1 \%$ penicillin/streptomycin, $50 \mathrm{ng} / \mathrm{ml}$ fungizone, and digested at $37^{\circ} \mathrm{C}$ while shaking with $0.3 \%$ collagenase type IA (Sigma-Aldrich) in DMEM/F12 by repeating cycles of 30 minutes until digestion was complete. Cell suspension was filtered through a 70um-cell strainer (BD Biosciences, San Jose, CA, USA), spun at 300g for 5 minutes, washed twice in PBS containing 1\% penicillin/streptomycin, $50 \mathrm{ng} / \mathrm{ml}$ fungizone (Thermo Fisher Scientific). The isolated chondrocytes were resuspended and cultured at $37^{\circ} \mathrm{C}, 5 \% \mathrm{CO}_{2}$ in DMEM/F12 with GlutaMAX (Thermo Fisher Scientific) supplemented with $10 \%$ fetal bovine serum (FBS), $50 \mu \mathrm{g} / \mathrm{ml}$ ascorbic acid (Thermo Fisher Scientific), 1\% penicillin/streptomycin and $50 \mathrm{ng} / \mathrm{ml}$ fungizone.

\section{siRNA knockdown of Tmem251}

Two sets of pre-designed Silencer Select siRNA against rat Tmem251 (s179664: GACAGUUAUUUUCUUGAUAtt, and s179666: GGAUUGGAGUGGGAUUGUAtt) were obtained from Life Technologies ${ }^{\circledR}$ (Thermo Fisher Scientific). siRNA against Gapdh (4390849) was used as a positive control and scrambled siRNA (4390843) was used as negative control.

\section{Chondrocyte transfection with siRNAs}

Chondrocytes $\left(25000 / \mathrm{cm}^{2}\right)$ were cultured in 24-well plates and transfected with Tmem251 specific or control siRNA when $60 \%$ confluency was reached, as described.(Lui et al., 2016) Briefly, cells were washed with PBS $1 \mathrm{~h}$ prior to transfection with PBS and medium was changed to DMEM/F12 without any antibiotics. Chondrocytes were separately transfected with 40nM of negative control siRNA, two siRNAs against TMEM251 (s179664 or s179666) and GapdhsiRNA (Thermo Fisher Scientific) using Lipofectamine 3000 (Thermo Fisher Scientific) according to manufacturer's instructions. $16 \mathrm{~h}$ post transfection, chondrocyte culture medium was replaced back to DMEM/F12 with GlutaMAX supplemented with $10 \%$ FBS, 50 $\mu \mathrm{g} / \mathrm{ml}$ ascorbic acid, $1 \%$ penicillin/streptomycin, and 50ng/ml fungizone.

\section{Quantitation of gene expression by real time PCR}

Cell lysates were collected $48 \mathrm{~h}$ post-transfection in solution $\mathrm{C}$ (4M guanidine thiocyanate, $25 \mathrm{mM}$ sodium citrate $\mathrm{pH} 7,0.1 \mathrm{M} \beta$-mercaptoethanol). Total RNA was extracted using phenol, chloroform as previously described.(Spath et al., 2018) Total RNA (50ng) was reverse-transcribed into cDNA by Superscript Reverse Transcriptase III (Thermo Fisher Scientific). Expression of Tmem251, Alpl and Col2a1 was quantified by ABI Prism 7900 Fast Sequence Detector (Thermo Fisher Scientific) by using specific primers (Table S5) and SYBR green (Thermo Fisher Scientific). Data were normalized to 18S rRNA and relative expression was calculated by using the formula: $2^{-\Delta^{\prime \prime} \tau *} 10^{6}$, with $\Delta \mathrm{Ct}$ being target gene expression relative to $18 \mathrm{~S}$ ribosomal RNA.(Nilsson et al., 2007)

\section{Statistical analyses}

Logarithm of odds (LOD) scores for data of NMD02 were calculated using MLINK program of the Linkage 3.3 package.(Terwilliger \& Ott, 1994) Only the participating branch was included for the purpose of LOD score calculations.

The siRNA experiments were independently performed twice, and for each experiment we had triplicates for each condition. The values in the graphs were calculated and presented as average \pm standard error of the mean (SEM) of technical replicates. Significance of each siRNA-treated sample compared to scrambled 
control was calculated by unpaired T test, using GraphPad Prism 7.0 (GraphPad Software, Inc., La Jolla, CA, USA) using raw data for absorbance and qPCR.

\section{Proliferation and apoptotic cell death assessment}

Proliferation, apoptosis and RNA expression were investigated 48h post- transfection. Proliferation was assessed by colorimetric detection based on 5-bromo-2'-deoxyuridine (BrdU) incorporation (Roche, Mannheim, Germany), according to manufacturer's protocol. Briefly, cells were incubated with BrdU for $3 \mathrm{~h}$, and absorbance was read at 370nm (SpectraMax Plus 384 Microplate Reader, Molecular Devices LLC, San Jose, CA, USA) $48 \mathrm{~h}$ post- transfection. Cells incubated for few seconds with BrdU- were used as background control.

Apoptosis was measured 48h post- transfection by photometric detection of cytoplasmic histone-associatedDNA-fragments in cell lysates according to manufacturer's instructions (Roche). Briefly, cell lysates were loaded on to a streptavidin-coated plate and subsequently incubated with biotinylated anti-histone antibodies and anti-DNA antibodies conjugated with peroxidase (POD). Unbound antibodies were removed by washing. Substrate solution, tetramethyl-benzidine (TMB) (Roche), was added. The peroxidase activity in the immunocomplex was determined photometrically at $405 \mathrm{~nm}$.

\section{Results}

\section{Clinical features}

Family NMD02 (Figure 1A) had seven individuals while that of family ID01 had one child (Figure 1B) affected with Mucolipidosis II alpha/beta like syndrome (OMIM\#252500) (Table 1). Five affected individuals in family NMD02 were siblings and alive when the study was initiated. Two (III:5, III:6) of these affected individuals died during the course of the study. The parents and six siblings of the five patients were healthy with no obvious phenotype. The affected siblings had similar phenotypes (Figure 1C-E). However, the phenotype of the youngest affected individual was less severe as compared to that manifested by the older siblings, supporting a progressive course of the disorder (Table 1). Coarsening of facial features was more severe in the oldest siblings. Two affected individuals (III:5 and III:6) lost walking ability as the disease progressed and became bed ridden in late teens. They both died in their early twenties. Cognition of all affected individuals was average and the patients responded to questions and their speech was not impaired. Complete blood counts of individuals III:9 and III:11 detected mild anemia but no other abnormality (Table 1). Clinical or imaging investigations apart from skeletal radiography were not possible due to limited access to proper health care.

Family ID01 was identified in Iran with one affected girl with the onset of symptoms at the age of 6 months (Figure $1 \mathrm{~F}$ ). She had severe skeletal dysplasia, coarse facial features, developmental delay, short stature, craniosynostosis, kyphoscoliosis, hip joint subluxation, abnormal teeth, upper airway problem and motor impairment. Brain MRI showed thinning of corpus callosum and mild ventriculomegaly. Heart echocardiography documented a small ventricular septal defect. Biochemical tests for metabolic disorders showed an increase in Iduronate-2 sulfatase (Table 1).

Radiographs of individuals III:9 and III:11 (Figure 2A-B) in family NMD02 indicated severe skeletal dysplasia reminiscent of dysostosis multiplex. The long bones were severely affected with prominent metaphyseal regions and abnormal epiphyses. Pelvic radiograph of individual III:11 revealed small iliac wings with abnormal proximal hips. The skeletal anomalies were more severe in the older sibling, supporting a progressive nature of the disorder.

Radiographs of the affected child from family ID01 indicated severe skeletal dysplasia reminiscent of dysostosis multiplex. Multiple hemivertebrae, paddle shape ribs, hypoplasia and flattening of acetabular roof were also revealed by radiography (Figure 2C). Diaphyseal widening in humerus with hypoplastic epiphysis and hypoplastic genoid fossa was evident. Moreover, hip radiographs indicated short iliac wings and left hip dislocation. The child died at the age of 5 years due to respiratory failure.

\section{Genetic Analyses}


Fluorescently labeled microsatellite markers located close to the respective genes (Table S1) excluded linkage of the disorder toGNPTAB (OMIM\#607840), GNPTG (OMIM\#607838), IDUA(OMIM\#611542) and ARSB (OMIM\#611542) for family NMD02. Analyses of the filtered exome data revealed three novel homozygous exonic variants located on chromosome 14q32 (Table S6) which also segregated with the phenotype in family NMD02 (Figure 1-2). Only TMEM251 variant c.133C > T;p.(Arg45Trp) was predicted to be damaging by all five prediction programs and affected an amino acid which was fully conserved in the orthologous proteins (Figure 3A). This variant was absent in all public databases and from 400 ethnically matched control chromosomes. The known genetic causes of various forms of mucopolysaccharidosis and mucolipidosis were also excluded by analyses of exome data for family NMD02 (Table S1).

We identified five regions of shared homozygosity located on different chromosomes from the exome data of the two affected individuals (Figure S1). Sequencing of the variants located in these regions revealed that only variants present on chromosome 14q32 (Table S2, Table S6 and data not shown) segregated with the phenotype with a LOD score of 3.2 at $\vartheta=0$. Moreover, no variants were identified by Sanger sequencing in those exons which were located within chromosome 14q32 region of shared homozygosity (Figure S1) and had not been covered by exome sequencing (Table S2).

Analysis of the trio exome sequencing data of family ID01 revealed one homozygous insertion variant c.215dupA;p.(Tyr72Ter) in TMEM251 in the patient, for which the parents were heterozygous carriers (Figure 1 and 2). None of the other 23 individuals from the pedigree were found to be homozygous for the variant. Moreover, this variant was absent from all public databases. The variant identified in family NMD02 was deposited in LOVD (\#0000601066) and the variant identified in family ID01 was deposited in ClinVar (SCV000998897).

\section{TMEM251 and TMEM251P}

TMEM251, also known as C14orf109 is an uncharacterized gene located on chromosome 14q32.12. TMEM251 is highly conserved gene (0.69 Z score and 21.351GDI score) and the identity among orthologues and the presence of very few variants in controls points to evolutionary importance of the protein. The two isoforms, NM_001098621.1 and NM_015676.1 of the gene are encoded by alternatively spliced exons in mammals while the genomes of other vertebrates have sequences corresponding to only one of the shorter isoforms (Figure 3B). A processed pseudogene of TMEM251 long isoform, TMEM251P, which contains multiple stop codons in the corresponding reading frame is located on the reverse strand within intron 85 of ADGRV1 on chromosome 5:90326406 -90327386 (hg19) (Figure 3C). The pseudogene is present in one to two copies in all primate genomes examined and is absent from genomes of other vertebrates.

\section{TMEM251 expression and protein domains}

Analyses of human expressed sequence tag (EST) data suggested that both the long isoform and the short isoform are expressed in various tissues, with the short isoform preferentially expressed in the brain as compared to the long isoform. We obtained transcripts for both isoforms of TMEM251 from blood, which was the only source of human RNA available to us. We were able to amplify Tmem251 from cDNA libraries mouse tissues including heart, brain, liver and kidney. In zebrafish, higher expression of tmem251 mRNA is detected in the axis at early somitogenesis as compared to other regions starting from one cell to pectoral fin stage.(Thisse \& Thisse, 2014) Tmem251 is also expressed in ovine muscles and is upregulated in skeletal muscles of Callipyge lambs.(Vuocolo et al., 2007)

TMEM251 has a conserved domain of unknown function (DUF4583) corresponding to amino acids 40 to 167 (NP_001092091.1) in the 169 amino acid protein in the human long isoform (Figure 3B). The short isoform lacks 35 amino acids from the N-terminal (Figure 3B). TMEM251 is predicted to be a transmembrane protein with two alpha helices (Figure 3D). No signal peptide was detected in the protein sequence by computational analyses. The N-terminal and C-terminal are predicted to face the lumen. ProtFun 2.2 analyses predicted TMEM251 to be a transporter with high odds of 4.139.

The missense variant p.(Arg45Trp) (NP_001092091.1), or p.(Arg7Trp) (NP_056491.1) affects an amino acid 
located close to the first transmembrane segment at the amino terminal of both isoforms of the protein. The variant is predicted to result in the incorporation of the mutated amino acid tryptophan inside the membrane as part of the first transmembrane segment making it unavailable in the lumen (Figure 3D). This effect is similar to that predicted for an arginine to tryptophan variant pathogenic allele of TMEM230 in Parkinson's disease.(Deng et al., 2016) The frameshift variant c.215dupA;p.(Tyr72Ter) is predicted to truncate the protein in the non-transmembrane segment located between the two transmembrane regions which may give rise to a shortened protein which may be unstable. No protein may be produced at all if nonsense-mediated decay of the mutant transcript occurs.

\section{TMEM251 localizes to Golgi complex and Plasma membrane}

Online searches indicated that TMEM251 is likely to be localized to the Golgi complex in humans and mice. GFP-fused wild-type TMEM251 was observed to localize to plasma membrane and strongly colocalized with a marker of Golgi complex, Giantin, in transiently transfected Human Osteosarcoma cells (Figure 4A). GFP-fused p.Arg45Trp TMEM251 also localized to Golgi complex and cell membrane (Figure 4B). In contrast, both wild-type and mutant proteins did not co-localize with markers of Endoplasmic reticulum (PDI) (Figure 4C-D) or lysosome (LAMP1) (not shown). The expression of GFP-fused p.Arg45Trp TMEM251 was markedly reduced and punctate when compared to that of the wild-type protein (Figure 4B and D).

\section{Tmem251 knockdown induces chondrocyte dedifferentiation}

Two different siRNAs decreased TMEM251 expression by 2.6 fold $(\mathrm{p}<0.001)$ and 1.6 fold $(\mathrm{p}<0.01)$ respectively, 48hrs after transfection of rat primary chondrocytes, compared to chondrocytes transfected with scrambled siRNA (Figure 4E). To determine whether Tmem251 knockdown affects chondrocyte differentiation, we investigated the expression levels of chondrocyte differentiation markers, such as Alkaline phosphatase $(A l p l)$ and Collagen type 2 (Col2a1). Expression of chondrogenic markers was not altered in cells treated with scrambled siRNA. In contrast, Tmem251 knockdown did not affect Alpl expression (Figure $4 \mathrm{~F})$ but significantly reduced Col2a1 expression by 1.4 fold $(\mathrm{p}<0.05)$ and 2.2 fold $(\mathrm{p}<0.001)$ respectively for the two siRNAs tested (Figure 4G). Reduction of Col2a1 expression, but not Alpl, expression in Tmem251 knockdown cells, suggests that TMEM251 is important for chondrocytic, but not hypertrophic differentiation, and is perhaps particularly required for maintenance of the chondrocytic phenotype.(Ma et al., 2013)

\section{Tmem251 knockdown does not affect apoptosis or chondrocyte proliferation}

Tmem251 knockdown did not affect chondrocyte apoptosis 48hrs post-transfection (Figure 4H), indicating that increased apoptosis of chondrocytes may not be a major pathogenic mechanism in this condition. Moreover, chondrocyte proliferation was not significantly affected by Tmem251 knockdown (Figure 4I). In summary, the Tmem251 knockdown experiment demonstrated that TMEM251 is important for chondrocytic differentiation.

\section{Discussion}

We have identified two novel homozygous variants in TMEM251 as the likely cause of a complex progressive skeletal disorder leading to severe short stature and early mortality in two unrelated families. Our findings indicate that TMEM251 may have a metabolic role. The clinical course of disease in both families was suggestive of a metabolic multisystemic disorder which was supported by the widespread TMEM251 expression pattern. The affected individuals in the two families had striking phenotypic similarities to patients with mucolipidosis and mucopolysaccharidosis(Bonafe et al., 2015) specifically to Mucolipidosis II/III based on patient characteristics of short stature, dysostosis multiplex, severe physical handicap, facial dysmorphology and distended abdomens (Dr. Leroy, personal communication). However, variants in the causative genes for these disorders (GNPTAB or GNPTG) were excluded as cause of the phenotype. Interestingly, both GNPTAB and GNPTG localize to Golgi complex, as is the case for TMEM251. Studies are required to test whether the three proteins participate in the same cellular pathways. 
The unavailability of patients' cells for RNA or protein expression studies hinders the evaluation of variants effects in vivo. Brain and cardiac imaging studies in the Iranian patient suggested the pathogenic variants in this gene could be responsible for cardiac and brain abnormalities. The unavailability of abdominal and cardiac imaging and laboratory examinations for the Pakistani family however, precluded further investigations to unravel organ abnormalities, including hepatosplenomegaly and cardiac anomalies.

Our results indicate that the p.(Arg45Trp) variant could be a functional null allele while the p.(Tyr72Ter) may also be a loss of function allele. The decreased localization of p.(Arg45Trp) TMEM251 to plasma membrane as well as to the Golgi complex may be due to improper folding of the protein. This may halt its incorporation into the membranes or cause degradation of the mutant TMEM251. The reduction in chondrogenic marker Col2a1 after siRNA-induced knockdown of Tmem251 in rat primary chondrocytes suggests that TMEM251 may play a role in the differentiation of chondrocytes and thus have an important function in the development of cartilage and bones. Future studies of animal models with Tmem251 variants could elucidate the role of the encoded protein in skeletogenesis in more detail.

In conclusion, our findings implicate homozygous TMEM251 variants as the genetic cause of a severe skeletal dysplasia with features of a metabolic disorder. Identification of additional affected individuals due to TMEM251 variants will delineate the full spectrum of the disorder and expand phenotypic manifestations as well as the molecular mechanisms involved in the disease course. Continued research will elucidate the role of TMEM251 in human physiology.

\section{Supplemental Data description}

Supplementary data includes 6 tables and 1 figure.

\section{Acknowledgements}

We are highly grateful to the members of family NMD02 and ID01 for their participation and co-operation in the study. We thank Dr. JG Leroy for helpful comments. One author (NA) was a recipient of an indigenous and an IRSIP scholarship from Higher Education Commission, Pakistan. This study was supported by personal funds (NM), NIMAD research grant (940714) awarded to MAF, Swedish Research Council (OM), Academy of Finland (OM), Sigrid Jusélius Foundation (OM) and a grant from Koshish Foundation, USA $(\mathrm{SN})$.

\section{Data Availability}

Data will be available from corresponding author upon reasonable request.

\section{Web Resources}

https://www.ncbi.nlm.nih.gov/nucest

http://www.proteinatlas.org/search/tmem251

http://www.cbs.dtu.dk/services/ProtFun/

https://www.ebi.ac.uk/Tools/msa/clustalo/

http://www.insilicase.com/agile/AgileVariantMapper.aspx

\section{References}

Ballabio, A., \& Gieselmann, V. (2009). Lysosomal disorders: from storage to cellular damage. Biochimica et Biophysica Acta (BBA)-Molecular Cell Research, 1793 (4), 684-696.

Bonafe, L., Cormier-Daire, V., Hall, C., Lachman, R., Mortier, G., Mundlos, S., . . . Sillence, D. (2015). Nosology and classification of genetic skeletal disorders: 2015 revision. American Journal of Medical Genetics Part A, 167 (12), 2869-2892. 
Carr, I. M., Bhaskar, S., O'Sullivan, J., Aldahmesh, M. A., Shamseldin, H. E., Markham, A. F., . . . Alkuraya, F. S. (2013). Autozygosity mapping with exome sequence data. Human Mutation, 34 (1), 50-56. doi:10.1002/humu.22220

Chiu, J., March, P. E., Lee, R., \& Tillett, D. (2004). Site-directed, Ligase-Independent Mutagenesis (SLIM): a single-tube methodology approaching $100 \%$ efficiency in 4 h. Nucleic Acids Research, 32 (21), e174-e174.

Coman, D., Irving, M., Kannu, P., Jaeken, J., \& Savarirayan, R. (2008). The skeletal manifestations of the congenital disorders of glycosylation. Clinical Genetics, 73 (6), 507-515.

Deng, H. X., Shi, Y., Yang, Y., Ahmeti, K. B., Miller, N., Huang, C., . . . Siddique, T. (2016). Identification of TMEM230 mutations in familial Parkinson's disease. Nature Genetics, 48 (7), 733-739.

Eklund, E. A., \& Freeze, H. H. (2006). The congenital disorders of glycosylation: a multifaceted group of syndromes. NeuroRx, 3 (2), 254-263.

Krakow, D. (2015). Skeletal dysplasias. Clinics in Perinatology, 42 (2), 301-319.

Kranz, C., Basinger, A. A., Gucsavaş-Çalıkoğlu, M., Sun, L., Powell, C. M., Henderson, F. W., . . . Freeze, H. H. (2007). Expanding spectrum of congenital disorder of glycosylation Ig (CDG-Ig): sibs with a unique skeletal dysplasia, hypogammaglobulinemia, cardiomyopathy, genital malformations, and early lethality. American Journal of Medical Genetics Part A, 143 (12), 1371-1378.

Li, H., \& Durbin, R. (2009). Fast and accurate short read alignment with Burrows-Wheeler transform. Bioinformatics, 25 (14), 1754-1760.

Longo, S., Bollani, L., Decembrino, L., Di Comite, A., Angelini, M., \& Stronati, M. (2013). Short-term and long-term sequelae in intrauterine growth retardation (IUGR). The Journal of Maternal-Fetal 85 Neonatal Medicine, 26 (3), 222-225.

Lui, J. C., Garrison, P., Nguyen, Q., Ad, M., Keembiyehetty, C., Chen, W., . . . Barnes, K. M. (2016). EZH1 and EZH2 promote skeletal growth by repressing inhibitors of chondrocyte proliferation and hypertrophy.Nature Communications, 7 , 13685.

Ma, B., Leijten, J. C. H., Wu, L., Kip, M., van Blitterswijk, C., Post, J. N., \& Karperien, M. (2013). Gene expression profiling of dedifferentiated human articular chondrocytes in monolayer culture. Osteoarthritis and Cartilage, 21 (4), 599-603.

McKenna, A., Hanna, M., Banks, E., Sivachenko, A., Cibulskis, K., Kernytsky, A., . . . Daly, M. (2010). The Genome Analysis Toolkit: a MapReduce framework for analyzing next-generation DNA sequencing data.Genome Research, 20 (9), 1297-1303.

Mortier, G. R., Cohn, D. H., Cormier-Daire, V., Hall, C., Krakow, D., Mundlos, S., . . . Savarirayan, R. (2019). Nosology and classification of genetic skeletal disorders: 2019 revision. American Journal of Medical Genetics Part A, 179 (12), 2393-2419.

Naz, S., \& Fatima, A. (2013). Amplification of GC-rich DNA for high-throughput family-based genetic studies. Molecular Biotechnology, 53 (3), 345-350. doi:10.1007/s12033-012-9559-y

Nilsson, O., Parker, E. A., Hegde, A., Chau, M., Barnes, K. M., \& Baron, J. (2007). Gradients in bone morphogenetic protein-related gene expression across the growth plate. Journal of Endocrinology, 193 (1), 75-84.

Saenger, P., Czernichow, P., Hughes, I., \& Reiter, E. O. (2007). Small for Gestational Age: Short Stature and Beyond. Endocrine Reviews, 28 (2), 219-251. doi:10.1210/er.2006-0039

Sparks, S. E., \& Krasnewich, D. M. (2017). Congenital disorders of N-linked glycosylation and multiple pathway overview. InGeneReviews@[Internet] : University of Washington, Seattle. 
Spath, S. S., Andrade, A. C., Chau, M., Baroncelli, M., \& Nilsson, O. (2018). Evidence That Rat Chondrocytes Can Differentiate Into Perichondrial Cells. JBMR Plus, 2 (6), 351-361. doi:10.1002/jbm4.10056

Stanley, P. (2011). Golgi glycosylation. Cold Spring Harbor Perspectives in Biology, 3 (4), a005199.

Terwilliger, J. D., \& Ott, J. (1994). Handbook of human genetic linkage : JHU Press.

Thisse, B., \& Thisse, C. (2014). Fast release clones: a high throughput expression analysis. 2004. ZFIN Direct Data Submission (http://zfin. org) .

Vuocolo, T., Byrne, K., White, J., McWilliam, S., Reverter, A., Cockett, N. E., \& Tellam, R. L. (2007). Identification of a gene network contributing to hypertrophy in callipyge skeletal muscle.Physiological Genomics, 28 (3), 253-272. doi:00121.2006 [pii]

10.1152/physiolgenomics.00121.2006

Wang, K., Li, M., \& Hakonarson, H. (2010). ANNOVAR: functional annotation of genetic variants from high-throughput sequencing data.Nucleic Acids Research, 38 (16), e164-e164.

\section{Figure Legends}

Figure 1. Pedigrees for NMD02 and ID01 and segregation of identified variants.

1. Pedigree of family NMD02 from Pakistan. Black symbols denote individuals affected with the disorder while open symbols represent the unaffected individuals. Strikethrough denotes deceased individual. Genotypes for the TMEM251 variant c.133C > T; p.(Arg45Trp) of all participants are shown under the respective symbols. Asterisks mark the individuals whose samples were subjected to exome sequencing.

2. Pedigree of family ID01 from Iran. Black symbols denote individuals affected with the disorder while open symbols represent the unaffected individuals. Strikethrough denotes deceased individual. Genotypes for the TMEM251 variant c.215dupA; p.(Tyr72Ter) of all participants are shown under the respective symbols. Asterisks mark the individuals whose samples were subjected to exome sequencing.

3. Individuals III:11 and III:9 at ages of 9 and 13 respectively. Photographs show progressive coarsening of facial features, short stature and skeletal deformities. The younger child does not have facial dysmorphism but the older brother has mild coarsening of features, a short stature and prominent wrists.

4. Protruding abdomen of individual III:9.

5. Bed ridden individual III:5, who is now deceased, at the age of 22 years. Note the coarse facial features with broad nose and prominent lips. Arms and hands are short, wrist joints are prominent.

6. Photographs of the affected child from Iranian family ID01. Coarse facial features and deformity are evident.

Figure 2. Clinical features of family NMD02 and ID01.

1. X-rays of individual III:9 of NMD02 show widened ribs with normal shaped thorax. Thoracic and cervical vertebrae are of reduced height. Femur, tibia and fibula are irregularly shaped with abnormal epiphyses. Feet show poor mineralization, curving of the metatarsal bones with osteolytic appearance of the joints. Epiphyses are irregular and abnormal in shape. The first toe has a short distal phalange and osteolytic appearance on both sides.

2. X-rays of individual III:11 of NMD02. Thoracic and lower limb. X-rays indicate similar features as in individual III:9 but with milder changes. Additionally, irregularly shaped iliac wings and abnormal prominent hips are also obvious. Forearms are clearly abnormal with curved and abnormally shaped radius and ulna with distal osteolysis. Overall, radiographs indicate dysostosis multiplex.

3. X-rays of an affected child from Iranian family. Spine X-ray indicates scoliosis due to multiple hemivertebrae and paddle shape ribs. Pelvic X-ray shows hypoplasia and flattening of acetabular roof and bilateral hip dislocation with short iliac wings.

4. Variant trace of TMEM251 c.133C $>$ T; p. (Arg45Trp) at site of variation. The changed base is shown by an arrow, while the affected codon is underlined. 
5. Variant trace of TMEM251 c.215dupA; p.(Tyr72Ter) at the site of variation. The inserted base is shown by an arrow, while the affected codon is underlined.Figure 3. Multiple Sequence alignment and graphical representation of TMEM251.

1. CLUSTAL Omega multiple sequence alignment of TMEM251 with orthologues from diverse vertebrate species. The gaps in alignment are denoted by dashes. Identical amino acids in all orthologues are indicated by asterisks below the alignment. The colons and periods depict amino acids which are highly conserved or less conserved, respectively. The p.Arg 45 residue affected by the variant is boxed. The first amino acid of the short isoform of TMEM251 is depicted by a small horizontal bar on top of the Methionine residue.

2. Schematic representation of human TMEM251 . Both the long and short isoforms are depicted. Gray boxes show the coding exons while the untranslated regions are indicated by black bars. Intron is represented by a horizontal line. The short isoform of the gene encodes a protein which lacks 38 amino acids from the N-terminal which are present in the long isoform.

3. Illustration of the processed pseudogene of TMEM251 long isoform which is located on chromosome 5 within intron 85 of $A D G R V 1$ (- or antisense strand). The gray vertical lines denote mismatches to TMEM251 sequence.

4. Diagrammatic representation of TMEM251 as encoded by the long isoform, inserted in the lipid bilayer of plasma membrane. The two transmembrane regions are shown by shaded rectangles (amino acids 47-66 and 105-127, respectively). $\mathrm{N}$ and $\mathrm{C}$ denote the amino and carboxyl terminal of the protein, respectively. The positions of the wild-type protein with p.Arg45 (left panel) and the variant p. Trp45 (right panel) are depicted by arrows. Note that the variant is predicted to result in inclusion of amino acid 45 into the first transmembrane segment which will be inserted in the membrane (right panel).

Figure 4. Localization of wild-type and mutant TMEM251 proteins in Human Osteosarcoma cell line and knockdown of Tmem25 in rat primary chondrocytes.

1. Analysis of wild-type TMEM251 localization in cells transfected with GFP-fused TMEM251 and immunostaining of Golgi complex with antibody against Giantin (red) nuclei with DAPI (blue). Merged image (yellow) indicates co-localization of wild-type TMEM251 with Golgi marker.

2. Analysis of mutant TMEM251 localization in cells transfected with GFP-fused mutant TMEM251 and immunostaining of Golgi complex with antibody against Giantin (red) nuclei with DAPI (blue), merged image (yellow) indicates co-localization of mutant TMEM251 with Golgi marker.

3. Immunostaining of Endoplasmic Reticulum with anti-PDI antibody after transfection with wild-type TMEM251 merged image indicates separate localization of wild-type TMEM251 and ER marker.

4. Immunostaining of Endoplasmic Reticulum with anti-PDI antibody after transfection with mutant TMEM251, merged image demonstrate mutant TMEM251 does not localize to ER. Note the reduced and punctate expression of mutant TMEM251 as compared to wild-type TEM251. (Images were taken in green, red and blue channel, separately and then merged).

1. Transfection of rat chondrocytes with two siRNAs (siR1 and siR2) against Tmem251 reduced mRNA expression of Tmem251, as determined by quantitative PCR.

2. Alpl expression and

3. Col2a1 expression measured by quantitative PCR in transfected cells compared to scrambled control. Alpl expression was not significantly changed in siR2 transfected cells, whereas Col2a1 expression was reduced in cells transfected with either siR1 or siR2.

4. Apoptosis in rat chondrocytes is not altered by Tmem251 knock-down, as measured by detection of cytoplasmic histone-associated-DNA-fragments.

5. Proliferation of chondrocytes measured by BrdU incorporation indicates no effect on proliferation upon Tmem251 knockdown. All measurements were performed $48 \mathrm{~h}$ post- transfection. Bars indicate average \pm SEM of two independent experiments $\left(*, \mathrm{p}<0.05 ;{ }^{* * *}, \mathrm{p}<0.001\right.$ relative to the scrambled-siRNA treated cells). 
A

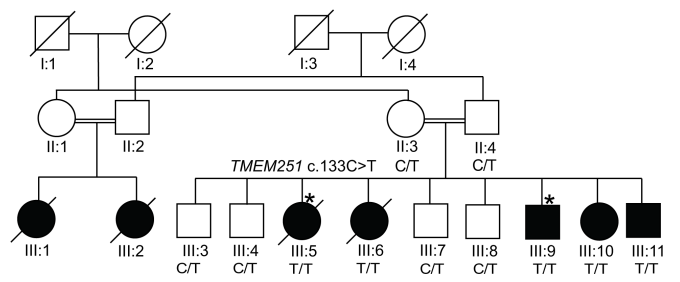

B

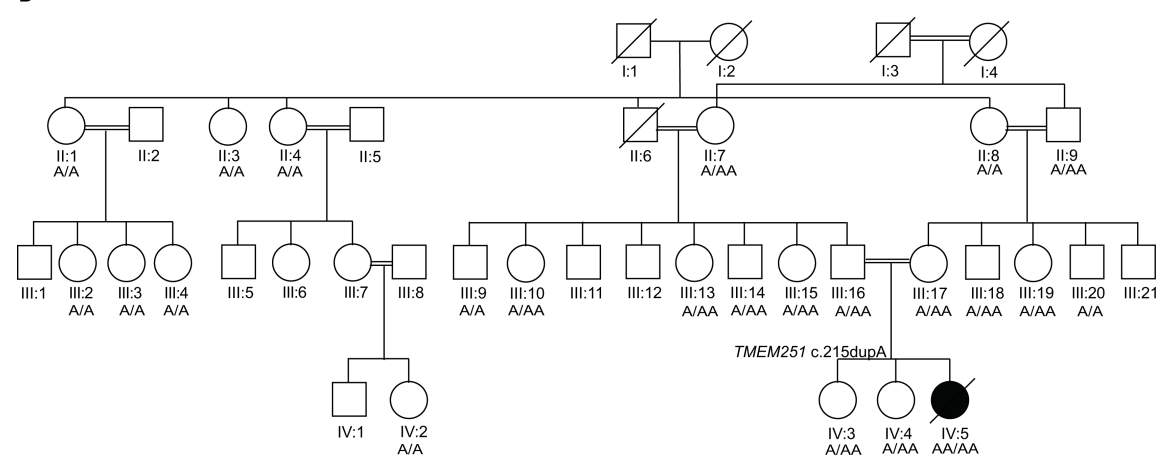

C
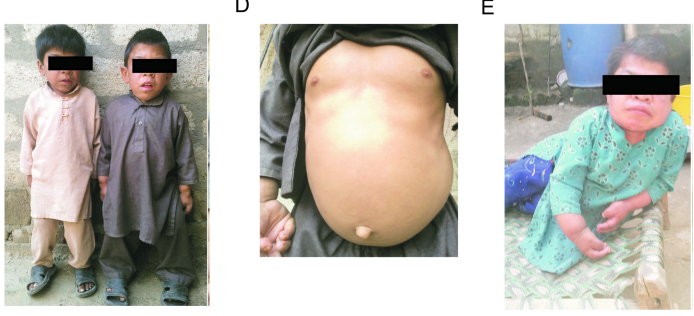

$\mathrm{F}$

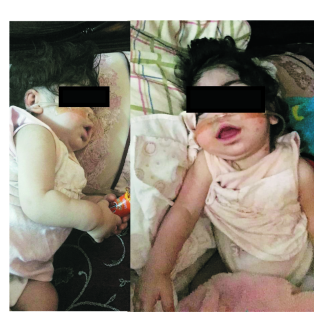

\section{Hosted file}

Table 1_Hum_mut.docx available at https://authorea.com/users/331838/articles/458366biallelic-tmem251-variants-in-patients-with-severe-skeletal-dysplasia-and-extreme-shortstature 

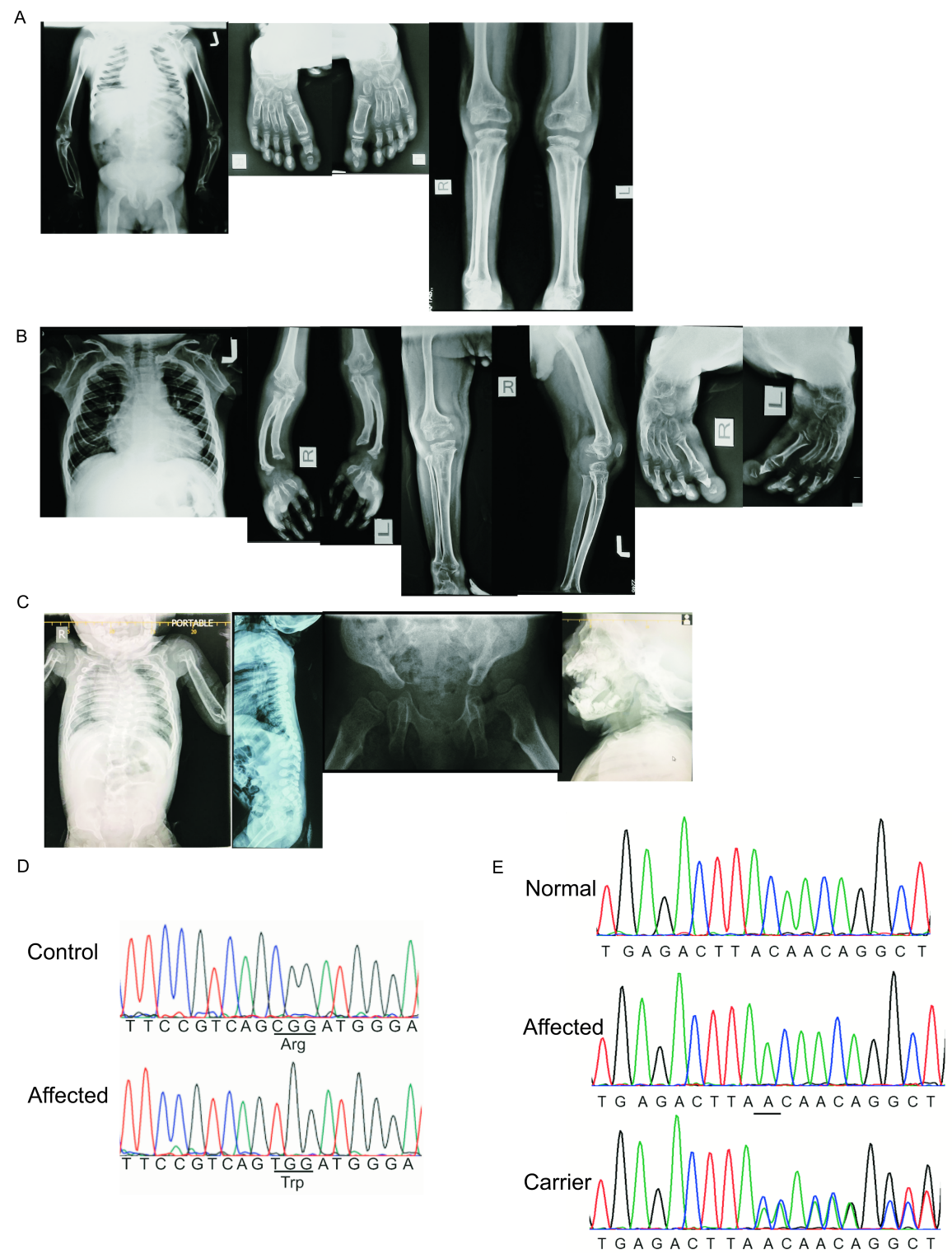
A

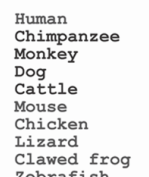

MLAFSEMPKPPDYSELSDSLTLAVGTGRFSGPLHRAWNMMNTRC RMGWIGVGLYLLASAA MLAFSEMPKPPDYSELSDSLTLAVGTGRF SGPLHRAWRMMNFR RRMGWIGVGLYLLASAA MVAFSEMPKPPDYSELNDSLTLAVGTGRF SGPLHRAWRMMNFR R WGWIGVGLYLLASAA -----MPKPPDYSELSDSLTLAVGTGRFSGPLHRAWRMMNFR R MGWIGVGLYLLASAA ----MPKAPDCSELSDSCSLAGGTGRF SGPLHRAWRMMNFR R MGWIGVGLYILASAA -

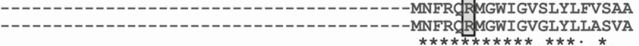

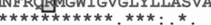

Human

Chimpanze

Dog

Cattle

Mouse

Zebrafish

AFYYVFEISETYNRLALEHIQQHPE-----------EPLEGTTWTHSLKAQLLSIPFWVW

AFYYYVEINETYNRLALEHIOOHPE---EPLETTWTHSLKAQLLSLPFWVW

AFYYVFEINETYNRLALEHIQQHPE---------EPLEGTTWTHSLKARLLSLPFWFW

AFYYVFEINETYNRLALEHIQQHPE-------EPLEGTTWTHSLKTRLLSIPFWFW

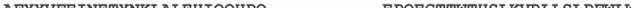

AFYYYVFEINETYNRLALEHIOOPPE---.-- KLKEETTWMHSLKTRLISSVPFWLW

AFYYVFE INETYNRLALEHVOLKPO----------EPHRGTTWTHSLKARLLSLPFWLW

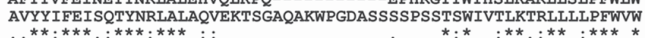

Human

Monkey

Dog

Cattle

Chicken

Lizard

Clawed frog
Zebrafish

TVIFLVPYLMFLFLYSCTRADPKTVGYCIIPICLAVICNRHQAFVKASNQISRIQLIDT TVIFLVPYLMFLFLLYSCTRADPKTVGYCIIPICLAVICNRHQAFVKASNQISRLQLIDT TIIFLVPYLQMFLFLYSCTRADPKTVGYCIIPICLAVICNRHQAFVKASNQISRLQLIDT TVIFLIPYLQMFLFLYSCTRADPKTVGYCIIPICLAVICNRHQAFVKASNQISRLQLIDT TITPLATS TIIFLIPYLOMFLFLYSCTRADPKTVGYCI IPICLAVICNRHOTFVKASNOISRLOLIDT TIIFLIPYLQMFLFLYSCTRADPKTVGYCIIPICLAVVCNRHQTFVKASNQISRLQLIDT AALFLIPYFQVFLFLYSCTRADPKTVGYCIIPICLAIICNRHQSFVKASNQISRLQLID ATIFLLPYLVFLFLYSRTRADPKTVGYCILPICLAVLCNRHQTFTKASNQISRIQLIDT

B
Chr5:90,326,406-90,327,386

(- strand )

TMEM251

TMEM251P

Long isoform

Short isoform

D
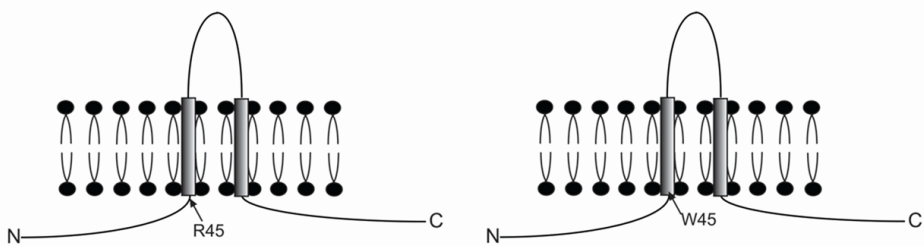


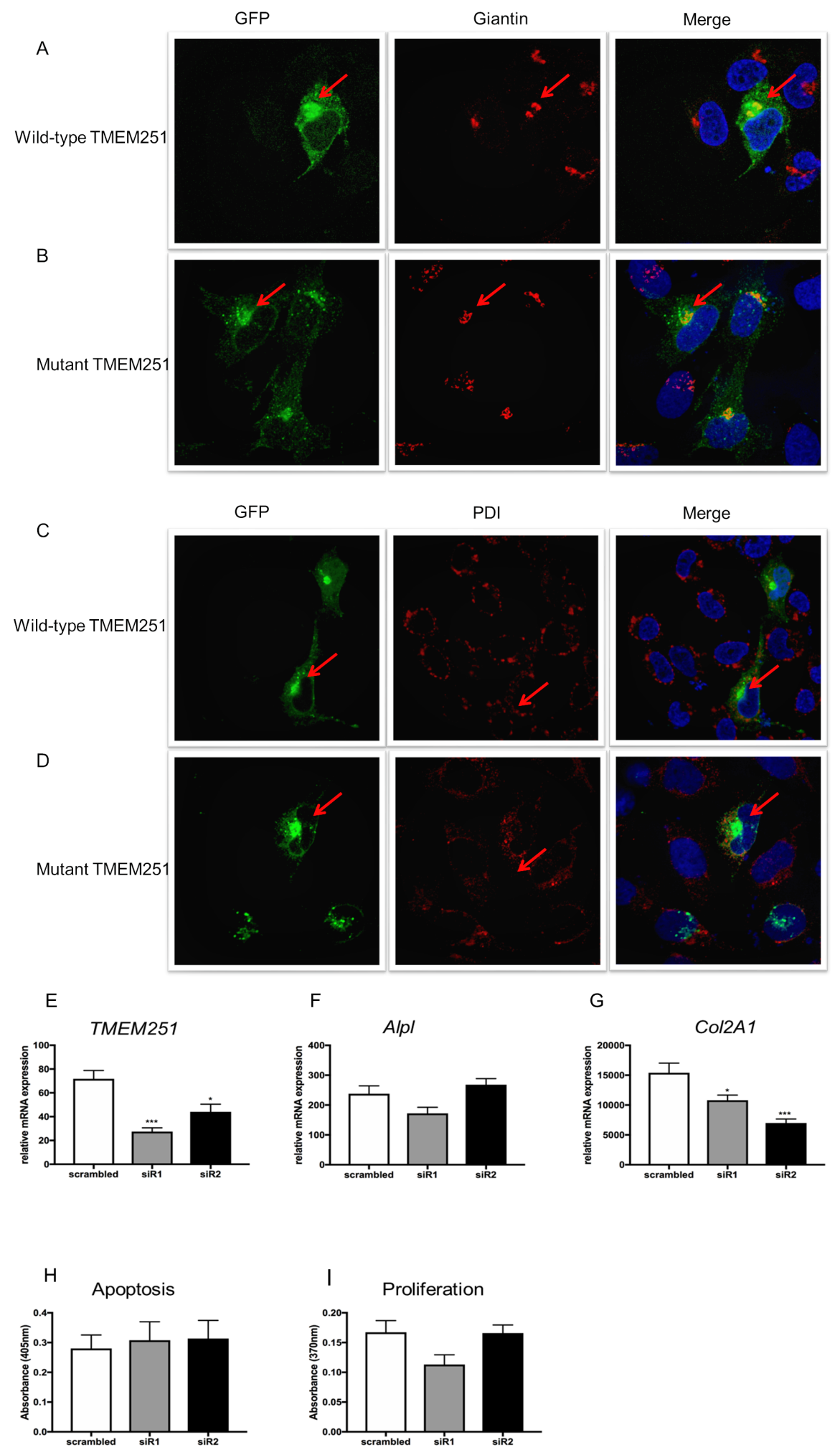

